\title{
On a Type of Semi-Symmetric Non-Metric Connection in HSU-Unified Structure Manifold
}

\author{
Shivani Sundriyal* and Jaya Upreti \\ (Communicated by Henrique F. De Lima)
}

\begin{abstract}
In the present paper, we have studied some properties of a semi-symmetric non-metric connection in HSU-unified structure manifold and HSU-Kahler manifold. Some new results on such manifolds have been obtained.
\end{abstract}

Keywords: Semi-symmetric non-metric connection; Levi-Civita connection; HSU-unified structure manifold; HSU-Kahler manifold; Nijenhuis tensor. AMS Subject Classification (2020): Primary: 53C25 ; Secondary: 53D15; 53B05; ; $53 B 15$.

\section{Introduction}

The idea of a metric connection on a Riemannian manifold was given by Hyden in 1932[6]. A linear connection $\nabla$ is said to be metric on a manifold $M^{n}$ if $\nabla g=0$; otherwise it is non-metric. In 1970, Yano[13] introduced semi-symmetric metric connection on Riemannian manifold. Smaranda[2], Agashe and Chafle[1], Sengupta[12], Chaubey[3][4] and many others [7][8][9][10][11] studied various and important properties of semi-symmetric metric and non-metric connections on several differentiable manifolds and also defined some new type of connections on Riemannian manifold.

Chaubey[5] studied a new type of semi-symmetric non-metric connection in 2019. He established that such connection on a Riemannian manifold is projectively invariant under certain conditions.

In the present paper, we have studied some properties of semi-symmetric non-metric connection defined in [5] on a HSU-unified structure manifold. Further, we also studied some properties of HSU-Kahler manifold with the same connection.

\section{Preliminaries}

Let $M^{n}$ be an even dimensional differentiable manifold of class $C^{\infty}$. Let there is a vector valued real linear function $\phi$ of differentiablity class $C^{\infty}$ satisfying

$$
\phi^{2} X=a^{r} X
$$

for some arbitrary vector field $X$. Also, a Riemannian metric $g$, such that

$$
g(\bar{X}, \bar{Y})=a^{r} g(X, Y)
$$

where $\bar{X}=\phi X ; 0 \leq r \leq n$ and $a$ is a real or complex number. Then $M^{n}$ is said to be HSU-unified structure manifold [11]. 
Now, let us define a 2-form $F$ in $M^{n}$ such that

$$
F(X, Y)=F(Y, X)=g(\bar{X}, Y)=g(X, \bar{Y})
$$

Then it is clear that,

$$
F(\bar{X}, \bar{Y})=a^{r} F(X, Y)
$$

from equation (2.3) it is clear that

$$
F(\bar{X}, Y)=a^{r} g(X, Y)
$$

The 2-form is symmetric in $M^{n}$. If HSU-unified structure manifold $M^{n}$ satisfies the condition

$$
\left(\nabla_{X} \phi\right) Y=0
$$

Then $M^{n}$ will said to be HSU-Kahler manifold.

From equation (2.6) it is clear that,

$$
\nabla_{X} \bar{Y}-\overline{\nabla_{X} Y} \Leftrightarrow \overline{\nabla_{X} \bar{Y}}=a^{r}\left(\nabla_{X} Y\right)
$$

where $\nabla$ is a linear Riemannian connection.

\section{A semi-symmetric non-metric connection}

Let $\left(M^{n}, g\right)$ be a Riemannian manifold of dimension $n$ endowed with a Levi-Civita connection $\nabla$ corresponding to the Riemannian metric $g$. A linear connection $\tilde{\nabla}$ on $\left(M^{n}, g\right)$ defined by [5]

$$
\tilde{\nabla}_{X} Y=\nabla_{X} Y+\frac{1}{2}\{\eta(Y) X-\eta(X) Y\}
$$

for arbitrary vector fields $X$ and $Y$ on $M^{n}$ is a semi-symmetric non-metric connection. The torsion tensor $\tilde{T}$ on $M^{n}$ with respect to $\tilde{\nabla}$ satisfies the equation

$$
\tilde{T}(X, Y)=\eta(Y) X-\eta(X) Y
$$

where $\eta$ is 1 -form associated with the vector field $\xi$ and satisfies,

$$
\eta(X)=g(X, \xi)
$$

and the metric $g$ holds the relation

$$
\left(\tilde{\nabla}_{X} g\right)(Y, Z)=\frac{1}{2}\{2 \eta(X) g(Y, Z)-\eta(Y) g(X, Z)-\eta(Z) g(X, Y)\}
$$

\section{HSU-unified structure manifold equipped with a semi-symmetric non-metric connection}

Theorem 4.1. Let $\left(M_{\tilde{\nabla}}^{n}, g\right)$ be a HSU-unified structure manifold. Then there exist a unique linear semi-symmetric non-metric connection $\tilde{\nabla}$ on $M^{n}$, given by equation (3.1) and satisfy equations (3.2) and (3.4).

Proof. Suppose $\left(M^{n}, g\right)$ is a HSU-unified structure manifold of dimension $n$ equipped with connection $\tilde{\nabla}$. Let $\tilde{\nabla}$ and Levi-Civita connection $\nabla$ are connected by the relation

$$
\tilde{\nabla}_{X} Y=\nabla_{X} Y+U(X, Y)
$$


for arbitrary vector fields $X, Y \in M^{n}$, where $U(X, Y)$ is a tensor field of type $(1,2)$. By definition of the torsion tensor $\tilde{T}$ of $\tilde{\nabla}$ and from equation (4.1) we have

$$
\tilde{T}(X, Y)=U(X, Y)-U(Y, X)
$$

so we have,

$$
g(\tilde{T}(X, Y), Z)=g(U(X, Y), Z)-g(U(Y, X), Z)
$$

from equations (3.2) and (4.3)

$$
g(U(X, Y), Z)-g(U(Y, X), Z)=\eta(Y) g(X, Z)-\eta(X) g(Y, Z)
$$

from eqution (3.4), we conclude that

$$
\left(\tilde{\nabla}_{X} g\right)(Y, Z)=-U^{\prime}(X, Y, Z)
$$

where $U^{\prime}(X, Y, Z)=g(U(X, Y), Z)+g(U(X, Z), Y)$.

Hence, by using equations (4.2), (4.3) and (4.5), we have

$$
g(\tilde{T}(X, Y), Z)+g(\tilde{T}(Z, X), Y)+g(\tilde{T}(Z, Y), X)=2 g(U(X, Y), Z)-U^{\prime}(X, Y, Z)+U^{\prime}(Z, X, Y)-U^{\prime}(Y, X, Z)
$$

Using equations (3.4) and (4.5) in equation (4.6), we have

$$
g(\tilde{T}(X, Y), Z)+g\left(\tilde{T}^{\prime}(X, Y), Z\right)+g\left(\tilde{T}^{\prime}(Y, X), Z\right)=2 g(U(X, Y), Z)-2 \eta(Z) g(X, Y)+\eta(X) g(Y, Z)+\eta(Y) g(X, Z)
$$

where

$$
g\left(\tilde{T}^{\prime}(X, Y), Z\right)=g(\tilde{T}(Z, X), Y)=\eta(X) g(Z, Y)-\eta(Z) g(X, Y)
$$

From equations (4.7) and (4.8) we get,

$$
U(X, Y)=\frac{1}{2}(\eta(Y) X-\eta(X) Y)
$$

and from equations (4.9) and (4.1) we have (3.1).

Conversely, we can show that if $\tilde{\nabla}$ satisfies equation (3.1), then it will also satisfy equations (3.2) and (3.4).

Hence, the theorem.

Theorem 4.2. On an n-dimensional HSU-unified structure manifold $\left(M^{n}, g\right)$ endowed with a semi-symmetric non-metric connection $\tilde{\nabla}$, the following relations hold;

(i) $\tilde{T}(\bar{X}, \bar{Y}, Z)+\tilde{T}(\bar{Y}, \bar{X}, Z)=0$

(ii) $\tilde{T}(\bar{X}, \bar{Y}, \bar{Z})+\tilde{T}(\bar{Y}, \bar{Z}, \bar{X})+\tilde{T}(\bar{Z}, \bar{X}, \bar{Y})=0$

(iii) $\tilde{T}(\overline{\bar{X}}, Y, Z)=\tilde{T}(X, \overline{\bar{Y}}, Z)=\tilde{T}(X, Y, \overline{\bar{Z}})$

(iv) $\tilde{T}(\overline{\bar{X}}, Y, Z)+\tilde{T}(\overline{\bar{Y}}, X, Z)=0$

(v) $\tilde{T}(\overline{\bar{X}}, Y, \overline{\bar{Z}})=a^{2 r} \tilde{T}(X, Y, Z)=\tilde{T}(X, \overline{\bar{Y}}, \overline{\bar{Z}})$

Proof. From equation (3.2) we have, $\tilde{T}(X, Y)=\eta(Y) X-\eta(X) Y$.

Also,

$$
\tilde{T}(X, Y, Z)=g(\tilde{T}(X, Y), Z)
$$

So that,

$$
\tilde{T}(X, Y, Z)=\eta(Y) g(X, Z)-\eta(X) g(Y, Z)
$$


Replacing $X$ by $\bar{X}$ and $Y$ by $\bar{Y}$ in above equation

$$
\begin{aligned}
& \tilde{T}(\bar{X}, \bar{Y}, Z)=\eta(\bar{Y}) g(\bar{X}, Z)-\eta(\bar{X}) g(\bar{Y}, Z) \\
& \tilde{T}(\bar{Y}, \bar{X}, Z)=\eta(\bar{X}) g(\bar{Y}, Z)-\eta(\bar{Y}) g(\bar{X}, Z)
\end{aligned}
$$

From equations (4.12) and (4.13), we get

$$
\tilde{T}(\bar{X}, \bar{Y}, Z)+\tilde{T}(\bar{Y}, \bar{X}, Z)=0
$$

Hence the result $(i)$.

Now, $\tilde{T}(\bar{X}, \bar{Y}, \bar{Z})=\eta(\bar{Y}) g(\bar{X}, \bar{Z})-\eta(\bar{X}) g(\bar{Y}, \bar{Z})$

Using equation (2.2),

$$
\tilde{T}(\bar{X}, \bar{Y}, \bar{Z})=a^{r} \eta(\bar{Y}) g(X, Z)-a^{r} \eta(\bar{X}) g(Y, Z)
$$

Similarly, we get,

$$
\begin{aligned}
& \tilde{T}(\bar{Y}, \bar{Z}, \bar{X})=a^{r} \eta(\bar{Z}) g(Y, X)-a^{r} \eta(\bar{Y}) g(Z, X) \\
& \tilde{T}(\bar{Z}, \bar{X}, \bar{Y})=a^{r} \eta(\bar{X}) g(Z, Y)-a^{r} \eta(\bar{Z}) g(X, Y)
\end{aligned}
$$

From equations (4.14),(4.15) and (4.16) we have the required result (ii).

Now,

$$
\tilde{T}(\overline{\bar{X}}, Y, Z)=\eta(Y) g(\overline{\bar{X}}, Z)-\eta(\overline{\bar{X}}) g(Y, Z)=a^{r} \eta(Y) g(X, Z)-a^{r} \eta(X) g(Y, Z)
$$

Hence, we have

$$
\tilde{T}(\overline{\bar{X}}, Y, Z)=a^{r} \tilde{T}(X, Y, Z)
$$

similarly,

$$
\begin{aligned}
& \tilde{T}(X, \overline{\bar{Y}}, Z)=a^{r} \tilde{T}(X, Y, Z) \\
& \tilde{T}(X, Y, \overline{\bar{Z}})=a^{r} \tilde{T}(X, Y, Z)
\end{aligned}
$$

From equations (4.17),(4.18) and (4.19) we have the required result (iii).

From equation (4.17), we can get (iv).

Now,

$$
\tilde{T}(\overline{\bar{X}}, \overline{\bar{Y}}, Z)=a^{2 r} \eta(Y) g(X, Z)-a^{2 r} \eta(X) g(Y, Z)
$$

Hence, we have

$$
\tilde{T}(\overline{\bar{X}}, \overline{\bar{Y}}, Z)=a^{2 r} \tilde{T}(X, Y, Z)
$$

similarly,

$$
\tilde{T}(X, \overline{\bar{Y}}, \overline{\bar{Z}})=a^{2 r} \tilde{T}(X, Y, Z)
$$

From euqtions (4.20) and (4.21), it is clear that result $(v)$ is also verified.

Hence, the theorem 4.2.

Theorem 4.3. A HSU-unified structure manifold $\left(M^{n}, g\right)$ endowed with a semi-symmetric non-metric connection $\tilde{\nabla}$, satisfies the following relations;

(i) $\left(\tilde{\nabla}_{X} \phi\right) Y=\left(\nabla_{X} \phi\right) Y+\frac{1}{2}\{\eta(\bar{Y}) X-\eta(Y) \bar{X}\}$

(ii) $\left(\tilde{\nabla}_{\bar{X}} \phi\right) \bar{Y}=\left(\nabla_{\bar{X}} \phi\right) \bar{Y}+\frac{1}{2}\left[a^{r}\{\eta(Y) \bar{X}-\eta(\bar{Y}) X\}\right]$ 
Proof. We have,

$$
\left(\tilde{\nabla}_{X} \phi\right) Y=\tilde{\nabla}_{X}(\phi Y)-\phi\left(\tilde{\nabla}_{X} Y\right)
$$

Using the equation (3.1) in equation (4.22), we get

$$
\left(\tilde{\nabla}_{X} \phi\right) Y=\tilde{\nabla}_{X}(\phi Y)-\phi\left(\nabla_{X} Y+\frac{1}{2}\{\eta(Y) X-\eta(X) Y\}\right)
$$

which implies,

$$
\left(\tilde{\nabla}_{X} \phi\right) Y=\left(\nabla_{X} \phi\right) Y+\frac{1}{2}\{\eta(\bar{Y}) X-\eta(Y) \bar{X}\}
$$

Hence, the result $(i)$.

Replacing $X$ by $\bar{X}$ and $Y$ by $\bar{Y}$ in result $(i)$, we get,

$$
\left(\tilde{\nabla}_{\bar{X}} \phi\right) \bar{Y}=\left(\nabla_{\bar{X}} \phi\right) \bar{Y}+\frac{1}{2}\left[a^{r}\{\eta(Y) \bar{X}-\eta(\bar{Y}) X\}\right]
$$

Hence the theorem.

Theorem 4.4. If a HSU-unified structure manifold $\left(M^{n}, g\right)$ admits a semi-symmetric non-metric connection $\tilde{\nabla}$, then the Nijenhuis tensor of Levi-Civita connection $\nabla$ and $\tilde{\nabla}$ coincide.

Proof. The Nijenhuis tensor with respect to $\phi$ is a vector valued bilinear function defined as, [7][10].

$$
\tilde{N}(X, Y)=[\bar{X}, \bar{Y}]-\overline{[\bar{X}, Y]}-\overline{[X, \bar{Y}]}+\overline{\overline{[X, Y]}}
$$

Since, for $X \in M^{n}, \overline{\bar{X}}=a^{r} X$. Hence,

$$
\tilde{N}(X, Y)=[\bar{X}, \bar{Y}]-\overline{[\bar{X}, Y]}-\overline{[X, \bar{Y}]}+a^{r}[X, Y]
$$

The Nijenhuis tensor with respect to Levi-Civita connection $\nabla$ is given by,

$$
N(X, Y)=\left(\nabla_{\bar{X}} \phi\right) Y-\left(\nabla_{\bar{Y}} \phi\right) X-\overline{\left(\left(\nabla_{X} \phi\right) Y\right)}+\overline{\left(\nabla_{Y} \phi\right) X}
$$

Using the result from theorem 4.3 , we have

$$
\left(\nabla_{X} \phi\right) Y=\left(\tilde{\nabla}_{X} \phi\right) Y-\frac{1}{2}\{\eta(\bar{Y}) X-\eta(Y) \bar{X}\}
$$

Replacing $X$ by $\bar{X}$ in equation (4.25)

$$
\left(\nabla_{\bar{X}} \phi\right) Y=\left(\tilde{\nabla}_{\bar{X}} \phi\right) Y-\frac{1}{2}\left\{\eta(\bar{Y}) \bar{X}-a^{r} \eta(Y) X\right\}
$$

Interchanging $X$ and $Y$ in equation (4.26)

$$
\left(\nabla_{\bar{Y}} \phi\right) X=\left(\tilde{\nabla}_{\bar{Y}} \phi\right) X-\frac{1}{2}\left\{\eta(\bar{X}) \bar{Y}-a^{r} \eta(X) Y\right\}
$$

Operating $\phi$ on both side of equation (4.25)

$$
\overline{\left(\nabla_{X} \phi\right) Y}=\overline{\left(\tilde{\nabla}_{X} \phi\right) Y}-\frac{1}{2}\left\{\eta(\bar{Y}) \bar{X}-a^{r} \eta(Y) X\right\}
$$

Interchanging $X$ and $Y$ in equation (4.28)

$$
\overline{\left(\nabla_{Y} \phi\right) X}=\overline{\left(\tilde{\nabla}_{Y} \phi\right) X}-\frac{1}{2}\left\{\eta(\bar{X}) \bar{Y}-a^{r} \eta(X) Y\right\}
$$

Put the value of equation $(4.26),(4.27),(4.28)$ and (4.29) in equation (4.24) we get

$$
N(X, Y)=\tilde{N}(X, Y)
$$

Hence, the theorem is proved. 


\section{HSU-Kahler manifold with a semi-symmetric non-metric connection $\tilde{\nabla}$}

As we discussed in section 2, that a HSU-unified structure manifold $M^{n}$ is said to be HSU-Kahler manifold if it satisfies the condition (2.6). That is;

$$
\left(\nabla_{X} \phi\right) Y=0
$$

In this section we will discuss some properties of HSU-Kahler manifold with a semi-symmetric non-metric connection $\tilde{\nabla}$.

Theorem 5.1. If $M^{n}$ be a HSU-Kahler manifold equipped with a semi-symmetric non-metric connection $\tilde{\nabla}$, then

(i) $\left(\tilde{\nabla}_{\bar{X}} \phi\right) \bar{Y}=\frac{a^{r}}{2}\{\eta(Y) \bar{X}-\eta(\bar{Y}) X\}$

(ii) $\left(\tilde{\nabla}_{X} \phi\right) Y=0$ iff $\eta(\bar{Y}) X=\eta(Y) \bar{X}$

Proof. From theorem 4.3 and equation (2.6), we have

$$
\left(\tilde{\nabla}_{X} \phi\right) Y=\frac{1}{2}\{\eta(\bar{Y}) X-\eta(Y) \bar{X}\}
$$

Replacing $X$ by $\bar{X}$ and $Y$ by $\bar{Y}$ in above equation, we have

$$
\left(\tilde{\nabla}_{\bar{X}} \phi\right) \bar{Y}=\frac{a^{r}}{2}\{\eta(Y) \bar{X}-\eta(\bar{Y}) X\}
$$

Hence, the result ( $i$ ). From equation (5.1) it is obvious that result (ii) will hold good in both sides.

Theorem 5.2. A HSU-Kahler manifold $M^{n}$ with a semi-symmetric non-metric connection $\tilde{\nabla}$ satisfies the following relation

$$
d F(X, Y, Z)=0
$$

Proof. We know that

$$
d F(X, Y, Z)=\left(\tilde{\nabla}_{X} F\right)(Y, Z)+\left(\tilde{\nabla}_{Y} F\right)(Z, X)+\left(\tilde{\nabla}_{Z} F\right)(X, Y)
$$

From equation (2.3) we have

$$
F(Y, Z)=g(\bar{Y}, Z)
$$

Differentiating (5.3) covariantly with respect to $X$ we get

$$
\tilde{\nabla}_{X} F(Y, Z)=\tilde{\nabla}_{X} g(\bar{Y}, Z)
$$

This implies,

$$
\left(\tilde{\nabla}_{X} F\right)(Y, Z)+F\left(\tilde{\nabla}_{X} Y, Z\right)+F\left(Y, \tilde{\nabla}_{X} Z\right)=\left(\tilde{\nabla}_{X} g\right)(\bar{Y}, Z)+g\left(\tilde{\nabla}_{X} \bar{Y}, Z\right)+g\left(\bar{Y}, \tilde{\nabla}_{X} Z\right)
$$

Using the equation (3.4), (5.1) and (5.3), we get

$$
\left(\tilde{\nabla}_{X} F\right)(Y, Z)=\eta(X) g(\bar{Y}, Z)-\frac{\eta(Z)}{2} g(X, \bar{Y})-\frac{\eta(Y)}{2} g(\bar{X}, Z)
$$

Similarly,

$$
\begin{aligned}
& \left(\tilde{\nabla}_{Y} F\right)(Z, X)=\eta(Y) g(\bar{Z}, X)-\frac{\eta(X)}{2} g(Y, \bar{Z})-\frac{\eta(Z)}{2} g(\bar{Y}, X) \\
& \left(\tilde{\nabla}_{Z} F\right)(X, Y)=\eta(Z) g(\bar{X}, Y)-\frac{\eta(Y)}{2} g(Z, \bar{X})-\frac{\eta(X)}{2} g(\bar{Z}, Y)
\end{aligned}
$$

Put the values from (5.4),(5.5) and (5.6) in equation (5.2) we have the required result. 
Theorem 5.3. The Nijenhuis tensor with respect to a semi-symmetric non-metrc connection $\tilde{\nabla}$ in a HSU-Kahler manifold $M^{n}$ vanishes, i.e; the manifold is integrable over $\tilde{\nabla}$.

Proof. The Nijenhuis tensor with respect to the connection $\tilde{\nabla}$ is defined as,

$$
\tilde{N}(X, Y)=\left(\tilde{\nabla}_{\bar{X}} \phi\right) Y-\left(\tilde{\nabla}_{\bar{Y}} \phi\right) X-\overline{\left(\left(\tilde{\nabla}_{X} \phi\right) Y\right)}+\overline{\left(\tilde{\nabla}_{Y} \phi\right) X}
$$

Replacing $X$ by $\bar{X}$ in equation (5.1), we have

$$
\left(\tilde{\nabla}_{\bar{X}} \phi\right) Y=\frac{1}{2}\left\{\eta(\bar{Y}) \bar{X}-a^{r} \eta(Y) X\right\}
$$

Interchanging $X$ and $Y$ in equation (5.8)

$$
\left(\tilde{\nabla}_{\bar{Y}} \phi\right) X=\frac{1}{2}\left\{\eta(\bar{X}) \bar{Y}-a^{r} \eta(X) Y\right\}
$$

Operating $\phi$ on both sides of equation (5.1)

$$
\overline{\left(\tilde{\nabla}_{X} \phi\right) Y}=\frac{1}{2}\left\{\eta(\bar{Y}) \bar{X}-a^{r} \eta(Y) X\right\}
$$

Interchanging $X$ and $Y$ in above equation

$$
\overline{\left(\tilde{\nabla}_{Y} \phi\right) X}=\frac{1}{2}\left\{\eta(\bar{X}) \bar{Y}-a^{r} \eta(X) Y\right\}
$$

Putting values from equations (5.8), (5.9), (5.10), and (5.11) in equation (5.7), we get

$$
\tilde{N}(X, Y)=0
$$

Hence, the theorem is proved.

\section{References}

[1] Agashe, N.S.: A semi-symmetric non-metric connection on a Riemannian manifold. Indian J. pure appl. Math. 23, 399-409 (1992).

[2] Andonie, OC., Smaranda, D.: Certaines connexions semisymetriques. Tensor. 31, 8-12 (1977).

[3] Chaubey, S. K., Ojha, R.H.: On a semi-symmetric non-metric and quarter symmetric metric connexions. Tensor N.S. 70 (2), 202-203 (2008).

[4] Chaubey, S. K., Ojha, R.H.: On a semi-symmetric non-metric connection. Filomat. 26 (2), 269-275 (2012).

[5] Chaubey, S. K., Yildiz, A.: Riemannian manifolds admitting a new type of semisymmetric nonmetric connection. Turkish Journal of Mathematics. 43 (4), 1887-1904 (2019)

[6] Hyden, A.: Sub-Spaces of a Space with Torsion. Proceedings of London Mathematical Society. 2 (1), 27-50 (1932).

[7] Kobayashi, S., Nomizu, K.: Foundation of differential geometry, Vol. I and II. Interscience Publisher, London (1969).

[8] Kumar, S., Kandpal, D., Upreti, J.: On a HSU-unified Structure Manifold with a Recurrent Metric Connection. Journal of Computer and Mathematical Sciences. 8 (8), 366-372 (2017).

[9] Kumar, S., Upreti, J.: A new connection in an almost para-contact manifold. Journal of National Academy of Mathematics, Gorakhpur. 28, 42-52 (2014)

[10] MIshra, R.S.: Structures on a differentiable manifold and their applications. Chandrama Prakashan, 50-A Balrampur Hause, Allahabad, India (1984)

[11] Nivas, R., Agnihotri, A.: On HSU-unified Structure Manifold with a Quarter-symmetric Non-metric Connection. Bulletin of Mathematical Sciences and Applications. 3, 63-70 (2013).

[12] Sengupta, J., De, U.C, Binh, T.: On a type of semi-symmetric non-metric connection on a Riemannian manifold. Indian Journal of Pure and Applied Mathematics. 31 (12) 1659-1670 (2000).

[13] Yano, K.: On semi-symmetric metric connections. Revue Roumaine de Mathematiques Pures et Appliquees. 15 1579-1586 (1970). 


\section{Affiliations}

SHIVANI SUNDRIYAL

AdDRESS: S.S.J Campus, Kumaun University, Dept. of Mathematics, 263601, Almora-India. E-MAIL: shivani.sundriyal5@gmail.com

ORCID ID: 0000-0001-6195-2572

JAYA UPRETI

ADDRESS: S.S.J Campus, Kumaun University, Dept. of Mathematics, 263601, Almora-India.

E-MAIL: prof.upreti@gmail.com

ORCID ID:0000-0001-8615-1819 